\title{
Simulación de mejoras en el sistema productivo de una curtiembre basada en el mapeo de su cadena de valor
}

\author{
Simulation of improvements in the production system of a tannery based on the \\ mapping of its value chain
}

\author{
I.M Bedoya-Díaz iD ; V.L Chud-Pantoja ; A.M. Paredes-Rodríguez iD
}

\begin{abstract}
Resumen- En la actualidad, las organizaciones buscan constantemente el mejoramiento continuo en sus operaciones, por lo cual se enfrentan al reto de ser más eficientes en la utilización de sus recursos. De esta manera, en esta investigación se desarrolló una estrategia de mejoramiento basada en metodologías de Lean Manufacturing, que considera la aplicación de Value Stream Mapping -VSM en una cadena de valor multi-etapa, estableciendo cinco fases para el mejoramiento de los procesos de cadenas que consideran multiples etapas de producción, entre ellas la elección de la familia de productos, construcción de mapa actual, identificación de oportunidades de mejora, construcción del mapa futuro, simulación de los procesos y selección de la estrategia de mejoramiento. La herramienta se aplicó en una cadena del sector del cuero, donde los principales resultados obtenidos evidenciaron la subutilización de espacios en fábrica, tiempos perdidos por desplazamientos y movimientos innecesarios. La implementación y simulación del VSM fue esencial para la validación de las mejoras propuestas, que lograron alcanzar una mayor productividad y eficiencia, considerando la disminución en el tiempo de ciclo y lead time.
\end{abstract}

Palabras Claves - Lean Manufacturing, Mapeo de cadena de valor, Simulación, Productividad, Curtiembre.

Abstract - Currenly, organizations constantly seek continuous improvement in their operations, so they face the challenge of being more efficient with their resources. In this way, an improvement strategy based on Lean Manufacturing methodologies was developed in this research, which considers the application of Value Stream Mapping -VSM in a multi-stage value chain, establishing five phases for the improvement of chain processes that consider multiple stages of production, including the choice of the product family, construction of the current map, identification of improvement opportunities, construction of the future map, simulation of the processes and selection of improvement strategy. The tool was applied in a chain of the leather sector, where the main results were obtained that showed the underutilization of spaces in the factory, lost time due to unnecessary movements and displacements. The implementation and simulation of the VSM was essential for the validation of the proposed improvements, which managed to achieve greater productivity and efficiency, considering the decrease in cycle time and lead time.

Este documento fue enviado el 17 de mayo del 2020 y fue aceptado el 29 de septiembre del 2020.

I.M. Bedoya-Díaz estuvo en la Universidad del Valle, sede Buga, Carrera 13 \#5-1 (ingrith.bedoya @ correounivalle.edu.co)

V.L Chud-Pantoja está ahora en la Universidad del Valle, sede Zarzal, Calle 14 \#7-134. (vivian.chud@ correounivalle.edu.co)
Index Terms —Lean Manufacturing, Value Stream mapping., Simulation, Productivity, Tannery.

\section{INTRODUCCIÓN}

T a implementación de metodologías Lean para el Ldiagnóstico de deficiencias en la cadena de valor resulta ser de vital importancia para lograr buenos resultados en el desempeño operacional de las industrias [1], puesto que representa el punto de partida para el inicio de ajustes y mejoras en la misma.

Muchas de las industrias manufactureras carecen de un método adecuado para la solución de problemas en sus procesos productivos; la gran mayoría soluciona dichos problemas de manera superficial generando a corto plazo el resurgimiento de los mismos con consecuencias peores. Afortunadamente, Lean Manufacturing como filosofía, ofrece un rendimiento superior para clientes, empleados, accionistas y sociedad en general, brindando un conjunto de herramientas para la solución de problemas de manera sistemática, de modo que estos se puedan erradicar desde su raíz por medio del rediseño de un sistema productivo que tiene como objetivo la minimización de desperdicios a través del flujo continuo de toda la cadena de valor [2] [3].

En la actualidad, las organizaciones presentan una dificultad para ver el flujo de valor de punta a punta y diagnosticar las causas sistémicas de los desperdicios que en él se encuentran [4]. Ante esta situación, el Mapeo de la Cadena de Valor (VSM, por sus siglas en inglés) se presenta como una técnica gráfica, útil y aplicable, que sirve como base para la propuesta de mejoras en sistemas productivos, ya que permite mapear las actividades con y sin valor agregado necesarias para llevar una familia de productos desde materia prima a producto terminado, con el fin de identificar oportunidades de mejora, y así, visualizar, plantear y dibujar un estado futuro, para la posterior ejecución de un plan estratégico con base en las mejoras

A.M Paredes-Rodríguez está ahora en Corporación Universitaria Minuto de Dios, sede Buga, Calle 21 sur Carrea 12 (aparedesrod@uniminuto.edu.co) 
identificadas, interviniendo de manera global en la línea de producción [5].

Diferentes estudios demuestran la utilidad de la aplicación de la herramienta VSM en diversos escenarios. Por ejemplo, en [6] aplican VSM en una empresa de producción de excavadoras, la cual presenta problemas en la línea, que impiden que la tasa de producción sea igual al takt time (ritmo al que se debe producir para cumplir con la demanda). Inicialmente los autores realizan un mapa de cadena valor actual para identificar aquellos inconvenientes en la línea, entre los que se puede destacar las averías de máquinas, la escasez de inventario en algunas estaciones y la falta de disponibilidad de herramienta. Luego, proceden a establecer unos kaizen de mejora al proceso, los cuales son simulados en el mapa de cadena de valor futuro. Entre los principales resultados de la investigación se encuentra una reducción de 156 minutos de las actividades de valor agregado que sincrónicamente reduce 430 minutos de actividades sin valor agregado, lo que contribuye a una disminución de 586 minutos del tiempo de producción actual.

Por su parte, [7] realizan un mapa de cadena de valor dinámico en tiempo real para almacenar, analizar y visualizar el comportamiento de un determinado proceso de producción en una pequeña y mediana empresa considerando el flujo de material, la eficiencia de la máquina y el rendimiento laboral. Esta herramienta permite a la compañía tomar decisiones rápidas y eficientes sobre los procesos de fabricación que no están agregando valor al producto. Asimismo, [8] elaboran una herramienta de toma decisiones empresariales que incluye la minería de datos sobre procesos logísticos internos unidos a un mapa de cadena de valor, con el objetivo de que pueda ser utilizado por cualquier compañía para identificar desperdicios y derivar recomendaciones para la acción. La herramienta es probada en una empresa que se dedica a la fabricación de automóviles, donde se tuvieron en cuenta 7500 partes y 15 procesos de referencia. Entre los principales resultados encontrados se resalta la identificación de un tiempo de espera prolongado de ciertas piezas críticas y una baja capacidad de rastreo de algunos procesos.

Del mismo modo, en [9] utilizan VSM como una herramienta para medir los desperdicios generados en la cadena de suministro de la leche en Uganda. Durante todo el proceso, de principio a fin, las pérdidas de alimentos a nivel de cadena se estiman en una magnitud de hasta el $14 \%$, lo que se traduce en un valor nutricional sustancial que desaparece del sistema alimentario, comprometiendo la capacidad de las personas para cumplir con sus recomendaciones nutricionales.

También, en [10] implementan la metodología mapeo de cadena de valor en una empresa que se dedica a la fabricación de bolsas de plástico, con el objetivo de identificar los recursos que no agregan valor y las estaciones cuellos de botella. Tanto el mapa de estado actual como el futuro se simulan y analizan en busca de diferentes atributos, como los tiempos de Takt, los plazos de producción y el tiempo de procesamiento para evaluar la ganancia general obtenida mediante la implementación de las mejoras en los procesos (kaizen). Los resultados de la simulación predijeron una reducción en el tiempo Takt de 46 a
26.6 minutos, un incremento en el número de rollos realizados por día de 28 a 50 y un aumento del tiempo de valor agregado en un $74.5 \%$.

Por otro lado, en [11] utilizan un enfoque integrado para cosechar los beneficios de las oportunidades de mejora identificadas a través de VSM y sus efectos en la métrica de efectividad total de los equipos (OEE, por sus siglas en inglés). Con este objetivo, generan una herramienta que ayuda a identificar cuellos de botella y a tomar decisiones acertadas para elegir el mejor método y la mejor herramienta para identificar las oportunidades de mejora. Los autores logran concluir que el uso de su propuesta en las empresas puede integrar principios y herramientas lean para obtener mejores resultados.

Una evidencia empírica sobre cómo la implementación de las prácticas de producción Lean se asocia con mejoras en el desempeño operativo de las empresas brasileñas de la cadena de suministro automotriz se presenta en [12], a través del estudio de 64 empresas de este sector. Los autores concluyen que las empresas de esta cadena han experimentado una reducción en el tiempo de entrega debido a la implementación de prácticas de mantenimiento total productivo (TPM, por sus siglas en inglés) y la reducción de inventario debido al uso de prácticas de justo a tiempo.

De igual forma, [13] explican la implementación de técnicas de lean manufacturing en un sistema de manufactura automotriz localizado al sur de la India. Después de la aplicación de las mejoras detectadas con el VSM, la compañía logró reducir el tiempo de fabricación en $40 \%$ ya que se redujeron los defectos dentro del proceso y se logró ampliar la capacidad de la planta, logrando una respuesta rápida a la demanda del cliente. Igualmente, en [14] aplican VSM a una planta que se dedica a fabricar repuestos del sector automotriz debido a que es una técnica lean que le ayuda a contrastar escenarios antes y después de la aplicación de mejoras a través de la medición de indicadores de gestión como lo son el tiempo de fabricación y la cantidad de inventario en proceso.

Por su parte, [15] presenta una metodología que integra el desarrollo sostenible con el mapeo de cadena de valor, esto con el objetivo de identificar las mejores estrategias y examinar aquellas prácticas recomendadas para mejorar el desempeño de algunos criterios de sostenibilidad. La herramienta es validada por un caso de estudio de la vida real aplicado en una empresa manufacturera de discos para satélite. Asimismo, [16] elaboran un VSM sostenible mediante la identificación de las métricas adecuadas y métodos para visualizarlos, enfocándose no solamente al impacto ambiental y económico sino también a la dimensión social; todo esto con el objetivo de desarrollar mejores estrategias para la producción sostenible.

En [17] se presenta la implementación de la técnica lean VSM en una empresa de tecnología de la información, debido a que esta herramienta es poco utilizada y tiene una aplicabilidad limitada en este sector. Los logros de la implementación son la reducción del tiempo de entrega, el tiempo de ciclo y los recursos utilizados. Los hallazgos de la investigación concluyen que, con el nuevo cambio de proceso, el tiempo total de entrega 
puede reducirse de 20 días a 3 días lo que equivale a un $92 \%$ de reducción en el tiempo de entrega global para el proceso de aprovisionamiento de bases de datos.

Por otro lado, [18] buscan mejorar el servicio de una empresa que ofrece servicios telefónicos sencillos, empleando transmisión de señal análoga mediante cable de cobre. La metodología utilizada por los autores involucra la implementación de VSM y un análisis de la cadena de valor con el objetivo de identificar aquellas actividades que agregan o disminuyen valor al proceso. Entre los resultados obtenidos se encuentra la disminución del tiempo de servicio en un 50\%, lo que habilita capacidad a la compañía para atender al menos el doble de la demanda de los clientes actuales.

En definitiva, según los casos anteriormente presentados se evidencia una aplicación de la herramienta VSM y la generación de resultados positivos en el desempeño operacional de industrias de diferentes sectores por la implementación de la misma. Sin embargo, no se encontró en la búsqueda realizada una metodología desarrollada y aplicada al sector del cuero, siendo potencial para la obtención de mejoras.

De esta manera, se analizó el sector del cuero en la industria colombiana, con el fin de implementar la herramienta VSM, centrando especial atención en las deficiencias que generan la necesidad de mejorar diversos aspectos tanto a nivel macro como a pequeña escala. Desde hace algunas décadas se identificó que los problemas asociados a las curtiembres se ven relacionados con el contrabando, bajas en la demanda de producto nacional, capacidad instalada ociosa, lenta evolución tecnológica, deficiencia de capital y maquinaria, baja calidad de materias primas, deficiencias de la planeación y control de la producción, bajas en los precios y bajos márgenes de utilidad [19]. Teniendo en cuenta la problemática del sector, se propone el desarrollo de una metodología para la aplicación multietapa, se escogió una pequeña curtiembre para el diagnóstico de los problemas de su cadena de valor, y se propusieron mejoras en diversos escenarios y finalmente se realizó la simulación de las mismas para la toma de decisiones en el sistema productivo.

El presente artículo, se enfoca en la implementación de la herramienta Lean, Mapeo de la Cadena de Valor (VSM, Value Stream Mapping), con la cual se abordó como caso de estudio, el estado actual de una pequeña curtiembre colombiana, con el fin de diagnosticar y proponer mejoras al desempeño de su sistema productivo que le permitan disminuir desperdicios y actividades innecesarias que generan costos y no agregan valor.

\section{MetodologíA}

Se propuso una metodología multi-fase, basada en lo presentado por [20], en la cual se aplicaron cada uno de los pasos de la herramienta VSM para cada una de las etapas del proceso de producción de tal manera que se obtuvieran mejoramientos en cada una de ellas, que generara una mejora global del proceso.

En este sentido, se proponen 5 fases (Fig. 1), la primera corresponde la elección de la familia de productos que será mapeada en la organización, la segunda fase incluye la construcción del mapa actual y la identificación de oportunidades de mejora para cada una de las etapas del proceso de producción del producto o familia de producto seleccionada, en esta se hace necesario definir indicadores que permitan medir el desempeño del proceso. La tercera etapa busca definir oportunidades de mejora a partir de los problemas identificados en el diagnóstico inicial del proceso; para luego en la fase 4, simular las mejoras seleccionadas y por último diagramar el mapa de estado de futuro del proceso (fase 5).

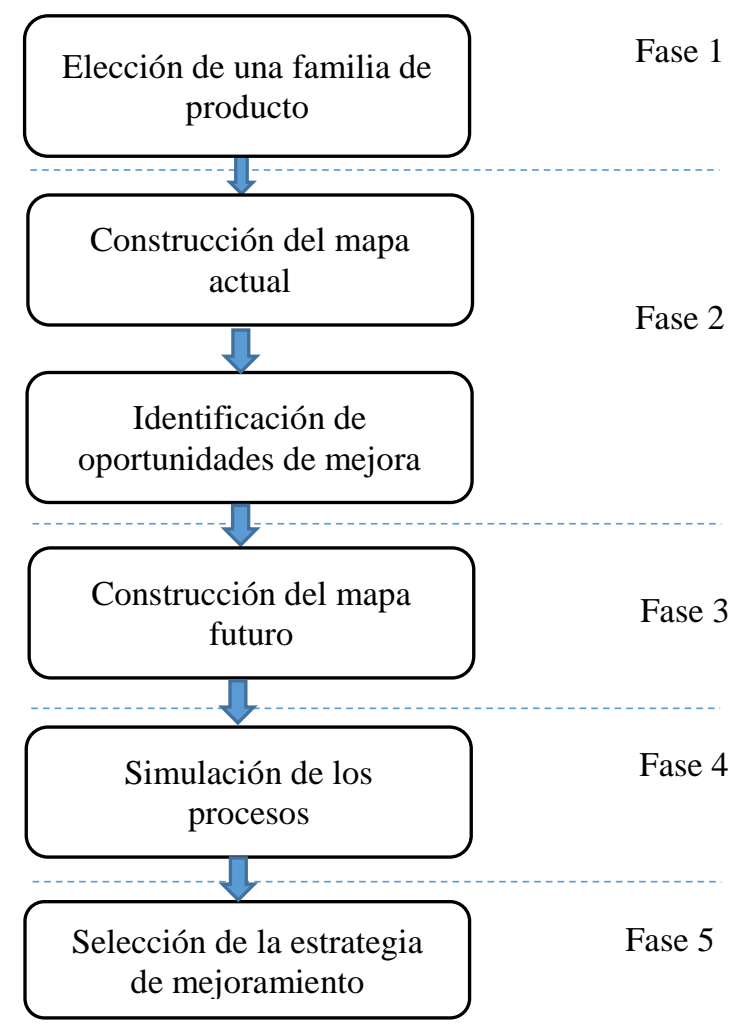

Fig. 1. Metodología propuesta para la aplicación de VSM en una cadena de valor multi-etapa

Fase 1

\section{A. Elección de una familia de producto.}

El VSM está enfocado en el análisis del flujo de valor de una familia de productos específica, por lo cual, las mejoras que se deriven de la implementación del mismo, deben ir encaminadas a agilizar el flujo de dicha familia. Una familia de productos se define como "un conjunto de productos que atraviesa por similares fases y aprovisionamiento común aguas abajo del proceso de fabricación antes de la entrega a su correspondiente cliente" [21]. En este sentido, la familia de productos que se selecciona para la implementación del VSM integra los ítems con un proceso de fabricación similar e identifica los problemas relacionados con la cadena de valor a tratar [2]. Para esta elección de la familia de producto que se analizará se deben realizar los pasos 1.1 y 1.2 , que se presentan a continuación. 


\section{1) Matriz de familia de productos}

Como herramienta de ayuda para la selección de familias de productos, diversos autores exponen la realización de una matriz [22]. Dicha matriz muestra la relación de todos los productos fabricados por la empresa con las operaciones por las cuales atraviesan, de modo que se pueden hallar fácilmente las similitudes entre las referencias de artículos que se consideren parte de la cadena de valor.

\section{B. Información de tiempos}

Los datos necesarios sobre la operación y los operarios, se deben registrar a través del estudio de tiempos y movimientos. De este modo, se identifica y comprueba si el método utilizado es el adecuado o el mejor en las circunstancias existentes [3]. Para completar la información de tiempos de las operaciones y construir el mapa del estado actual es necesario tener en cuenta los siguientes conceptos:

Takt time: También llamado tiempo de ritmo, se emplea para sincronizar el tiempo de producción con el de ventas. Es un número de referencia que da una sensación del ritmo al que hay que producir. Para lograr la correcta aplicación de este concepto es necesario realizar una revisión previa de los procesos, con el fin de lograr flexibilidad en todos los recursos empleados, dado que ante una variación del takt time, pueda regularse fácilmente [3].

$$
\text { Takt time }=\frac{\text { tiempo operativo por periodo en segundos }}{\text { Demanda del cliente por periodo en unidades }}
$$

Lead time: período de tiempo necesario para producir un sólo producto desde que el cliente hace su pedido, hasta que se le despacha.

Tiempo de ciclo: es la cantidad de tiempo necesario para que se complete la fabricación de una pieza hasta el momento en que la pieza siguiente se termina. Este tiempo debe coincidir con el transcurrido desde que el operario de la última operación del proceso se ocupa de esta actividad, hasta que la repita.

Tiempo de cambio de un proceso a otro: tiempo transcurrido desde que se completa la última pieza buena en operación de una sola referencia hasta el momento en que se completa la primera pieza de una referencia diferente [20].

Tiempo de Actividad (Uptime), o confiabilidad de los equipos: corresponde al porcentaje de tiempo que una pieza del equipo funciona correctamente sin reproceso, cuando es utilizada para una labor específica. Se calcula porcentualmente, del total del tiempo que se utiliza el equipo, cuanto funciona de manera adecuada [20].

Disponibilidad de los equipos: porcentaje de tiempo que un componente compartido entre varios flujos de valor se encuentra disponible para la fabricación de piezas en la cadena de valor que se le asigna [20].
OEE (Overall Equipment Efficiency o Eficiencia General de los Equipos): es una razón porcentual que sirve para medir la eficiencia productiva de la maquinaria. Mide en un único indicador, tres parámetros fundamentales en la producción industrial: la disponibilidad, la eficiencia y la calidad.

Fase II

\section{B. Construcción del mapa actual.}

Una vez seleccionada la familia de productos, comienza la construcción del Mapa del estado actual que según [20], es la línea de base para ver todos los procesos existentes de los cuales se proponen y miden todas las mejoras. Esta actividad da como resultado la identificación de actividades que agregan o no valor, las que deben ser eliminadas y las que continúan en el proceso de construcción del VSM futuro.

Autores como [21] y [23] sugieren para la construcción del mapa actual los siguientes pasos:

Paso 1. Dibujar los clientes: Dibujar aguas arriba comenzando con los requerimientos del cliente, la demanda y su patrón.

Paso 2. Dibujar los procesos productivos: Se dibuja cada proceso por el cual fluye el material mediante una caja. Este se termina donde una desconexión hace que el flujo de materiales se interrumpa y genere un inventario de producto en proceso. En este punto, se registran los datos necesarios para cada proceso: Recurso compartido o específico a la familia, tiempo de ciclo, tiempo de cambio, eficiencia, periodo de ciclo de fabricación, tamaño de lote medido en tiempo, número de operarios, número de variaciones de productos, tipo y tamaño de embalaje, números de relevos, tiempo de trabajo disponible (descontando descansos), y otros datos que se puedan considerar necesarios.

Paso 3. Representar los puntos de inventario: Normalmente entre los procesos existen puntos donde se acumula el inventario y, por lo tanto, puntos donde el material deja de fluir, por lo cual es importante dibujar el inventario acumulado y contabilizado en la propia planta.

Paso 4. Dibujar el flujo de materiales: esta gráfica abarca el flujo de materiales desde el almacén de la planta hasta el cliente, representando las principales entregas a clientes y de los proveedores, con su frecuencia de entregas, volúmenes de entregas, tiempo de reposición, entre otros.

Paso 5. Dibujar los flujos de información: Después de graficar el flujo físico de materiales, se grafica el flujo de información, de tal modo que se logren integrar al análisis de toda la representación gráfica del VSM.

Paso 6. Dibujar la relación entre procesos: En este paso los iconos utilizados marcan la diferencia entre un modelo push o pull en la organización. Trabajar en modo "push" significa que un proceso no se preocupa de lo que necesita el proceso que viene más tarde, sino que produce y "empuja" los materiales hacia adelante creando inventario. Mientras que en el modo 
"pull" es el siguiente proceso que "estira" la producción del anterior, por lo que éste produce estrictamente lo que es necesario sin crear exceso de inventario.

Paso 7. Dibujar las líneas de tiempo: Se contabiliza todo el tiempo de trabajo del producto y su periodo de maduración desde materia prima hasta producto terminado, mostrándolo en una línea de tiempo en la parte inferior del gráfico [2].

\section{Fase III}

\section{Identificación de oportunidades de mejora}

De acuerdo con [20], el análisis para identificar las oportunidades de mejora en un proceso en estudio se compone de tres partes fundamentales, para las cuales es indispensable conocer claramente la cadena de valor desde el proveedor hasta el cliente:

El flujo de información: Indica la manera en que fluye la información desde que se recibe un pedido hasta que es entregado. Al representarlo, se debe describir claramente a través de qué procesos y bajo qué medios fluye la información, diferenciando si fluye de manera física o electrónica, señalando la frecuencia en que se reciben pedidos, en que se programa la producción y bajo qué metodología. Gráficamente, se representa con la interacción de cliente, proveedor y control de la producción en la parte superior del diagrama.

El flujo de materiales o producción: En esta parte se muestra la transformación física de las materias primas a producto terminado, aquí se deben considerar todas las operaciones, movimientos, inventario en proceso y almacenamiento de producto terminado. Este flujo se detalla gráficamente en una caja que corresponde a cada actividad, en ella se señala el número de operarios, el tiempo de ciclo, tiempo de operación, disponibilidad y capacidad. También, deben incluirse líneas que indican la dirección del flujo y el tipo de sistema en el que fluyen los materiales, ya sea Push o Pull.

Líneas de tiempo y distancia de viaje: En este punto se integran dos líneas, la superior mide los tiempos de espera de cada proceso, indicando el tiempo total que tarda la entrega de producción en cuanto a la fabricación, en esta línea se refleja si el tiempo empleado agrega valor o no. La línea inferior refleja la distancia recorrida a través de todo el proceso de fabricación, entre las diferentes operaciones, esta línea se ve afectada por la cantidad de desplazamientos y la reiteración de los mismos, al finalizar refleja la distancia recorrida total.

\section{Fase IV}

\section{Simulación de las mejoras propuestas.}

Una vez identificados los puntos que se deben mejorar y plasmados en un mapa de la cadena de valor futuro, se procede a crear escenarios de simulación en los cuales varíen los tiempos de ejecución, tiempos de ciclo, lead time y distancias recorridas, entre otros, permite evaluar la viabilidad del estado futuro y de esta manera, contribuir a la definición de un plan de acción para la implementación real de las mejoras propuestas.

Fase V

\section{E. Construcción del mapa futuro}

El mapeo del estado futuro, está relacionado con lo que se quiere lograr al eliminar las actividades que no agregan valor. Surge del análisis del mapa de estado actual y se centra en mostrar las mejoras y los resultados obtenidos con las mismas, en este se debe incluir los cambios con relación a centros de trabajo, operaciones, flujo de materiales, metodologías de manejo de inventario, implementación de Kanban, mejoras en la programación de la producción y el flujo de la información.

Según [21] el VSM futuro es la proyección del estado ideal a la realidad. Para construirlo y graficarlo sugiere responder una serie de preguntas relacionadas con los indicadores y directrices de la filosofía Lean:

1) ¿Cuál es el takt time o ritmo impuesto por el mercado basado en el tiempo de trabajo disponible?

2) ¿Se va a producir para expedir directamente a partir del proceso regulador o se necesita un supermercado de producto terminado?

3) ¿Dónde puede ser empleado el flujo continuo?

4) ¿Es necesario algún sistema pull para gestionar la producción?

5) ¿Qué único punto de la cadena de producción, denominado proceso regulador será empleado para programar la producción?

6) ¿Cómo puede ser nivelado el mix de producción en el proceso regulado?

7) ¿Qué unidad de trabajo será retirada constantemente desde el proceso regulador?

8) ¿Qué mejoras de proceso (mejoras kaizen) serán necesarias en el flujo de valor para que fluya como en el diseño del estado futuro?

Los cuestionamientos planteados, se basan en las directrices de Lean Manufacturing, que como resultado, buscan que cada proceso produzca justamente lo que el siguiente proceso necesita, en el momento que este lo requiera, con el fin de unificar los procesos en un flujo continuo con un mínimo de periodo de maduración, un bajo costo y un máximo de calidad de producto [2].

\section{F. Selección de la estrategia de mejoramiento}

Esta fase corresponde a la elaboración y aprobación de un cronograma con un plan de acciones a partir de las mejoras aceptadas y validadas en la simulación del estado futuro. En teoría no debería haber discusión sobre el contenido de los diferentes proyectos a desarrollar, ya que estos han sido previamente admitidos con la finalización del mapa futuro. En [21] se aconsejan dividir el mapa futuro en segmentos manejables para emprender lentamente los proyectos de mejora. El segmento inicial podría ser aquel que integra al proceso regulador, o aquel proceso que es completamente 
comprendido por el personal de la empresa y tenga éxito e impacto garantizado.

\section{RESUltados OBtENIDOS}

La empresa objeto de estudio es una curtiembre que lleva en el sector de cuero en Colombia aproximadamente 17 años. El sistema productivo opera bajo pedido y con un pronóstico empírico de dos meses. La organización actualmente comercializa 12 productos generales de cuero, cuya producción se hace a través de cuatro procesos principales. En la Fig. 2 se muestra el esquema de producción general de la empresa, en donde se exhibe las actividades principales de cada proceso

\begin{tabular}{|c|c|c|c|}
\hline Ribera & Curtido & Recurtido & Acabado \\
\hline $\begin{array}{l}\text { - Recepción de } \\
\text { materia prima } \\
\text { - Pretratamiento } \\
\text { - Remojo } \\
\text { - Pelambre } \\
\text { - Descarnado } \\
\text { - Dividido }\end{array}$ & $\begin{array}{l}\text { - Desencalado } \\
\text { - Rendido } \\
\text { - Desengrasado } \\
\text { - Piquelado } \\
\text { - Curtido } \\
\text { - Rebajado }\end{array}$ & $\begin{array}{l}\text { - Recurtido } \\
\text { - Ablandado } \\
\text { - Esmerilado }\end{array}$ & $\begin{array}{l}\text { - Pintura } \\
\text { - Prensado y } \\
\text { recorte } \\
\text { - Desorille }\end{array}$ \\
\hline
\end{tabular}

Fig. 2. Procesos generales de la curtiembre

\section{A. Elección de una familia de producto.}

El portafolio de productos de la empresa está compuesto por doce clasificaciones generales de cuero, de las cuales seis comparten entre el noventa y el cien por ciento de las operaciones realizadas en todo el proceso. Teniendo como principal criterio la participación operativa, se seleccionó la familia de productos correspondiente al cuero de origen caprino a pesar de su inferior participación sobre los ingresos, ya que esta cuenta con un importante posicionamiento debido a la exclusividad y variedad de sus productos. Luego se procedió a analizar los procesos por los cuales pasaban este tipo de productos, con lo que se generó una matriz de familia de producto (ver tabla I). Se seleccionaron como productos para el estudio aquellos que compartían procesos y tenían una mayor representación en términos de ventas. Los artículos seleccionados fueron: capreto, consumato, bambi, cuero con pelaje, forros y gamos.

TABLA I.

MATRIZ FAMILIA- PRODUCTO DE ARTÍCULOS DE CUERO CAPRINO

\begin{tabular}{|c|c|c|c|c|c|c|c|c|c|c|}
\hline & \multicolumn{10}{|c|}{ "Operaciones } \\
\hline & $\begin{array}{c}\text { Pesaj } \\
\text { e y } \\
\text { clasif } \\
\text { icació } \\
\text { n }\end{array}$ & $\begin{array}{l}\text { C } \\
\text { or } \\
\text { te }\end{array}$ & $\begin{array}{l}\text { Re } \\
\text { mo } \\
\text { jo }\end{array}$ & $\begin{array}{c}\text { Pela } \\
\text { mbr } \\
\text { e }\end{array}$ & $\begin{array}{c}\text { Desc } \\
\text { amad } \\
0\end{array}$ & $\begin{array}{c}\text { Div } \\
\text { idid } \\
0\end{array}$ & $\begin{array}{c}\text { Dese } \\
\text { ncala } \\
\text { do }\end{array}$ & $\begin{array}{c}\text { Ren } \\
\text { did } \\
0\end{array}$ & $\begin{array}{c}\text { Desen } \\
\text { grasad } \\
0\end{array}$ & $\begin{array}{c}\text { Etiq } \\
\text { ueta } \\
\text { do }\end{array}$ \\
\hline $\begin{array}{l}\text { Capr } \\
\text { eto }\end{array}$ & $\mathrm{X}$ & $\mathrm{X}$ & $\mathrm{X}$ & $\mathrm{X}$ & $\mathrm{X}$ & $\mathrm{X}$ & $\mathrm{X}$ & $\mathrm{X}$ & $\mathrm{X}$ & $\mathrm{X}$ \\
\hline $\begin{array}{l}\text { Bam } \\
\text { bis }\end{array}$ & $\mathrm{X}$ & $\mathrm{X}$ & $\mathrm{X}$ & $\mathrm{X}$ & $\mathrm{X}$ & $\mathrm{X}$ & $\mathrm{X}$ & $\mathrm{X}$ & $\mathrm{X}$ & $\mathrm{X}$ \\
\hline $\begin{array}{l}\text { Forro } \\
\text { s }\end{array}$ & $\mathrm{X}$ & $X$ & $\mathrm{X}$ & $\mathrm{X}$ & $\mathrm{X}$ & $\mathrm{X}$ & $\mathrm{X}$ & $\mathrm{X}$ & $\mathrm{X}$ & $\mathrm{X}$ \\
\hline $\begin{array}{l}\text { Cons } \\
\text { umat } \\
\text { os }\end{array}$ & $\mathrm{X}$ & $\mathrm{X}$ & $\mathrm{X}$ & $\mathrm{X}$ & $\mathrm{X}$ & $\mathrm{X}$ & $\mathrm{X}$ & $\mathrm{X}$ & $\mathrm{X}$ & $\mathrm{X}$ \\
\hline $\begin{array}{l}\text { Gam } \\
\text { os }\end{array}$ & $\mathrm{X}$ & $\mathrm{X}$ & $\mathrm{X}$ & $\mathrm{X}$ & $\mathrm{X}$ & $\mathrm{X}$ & $\mathrm{X}$ & $\mathrm{X}$ & $\mathrm{X}$ & $\mathrm{X}$ \\
\hline
\end{tabular}

\begin{tabular}{|c|c|c|c|c|c|c|c|c|}
\hline $\begin{array}{l}\text { C. } \\
\text { Con } \\
\text { pelaj } \\
\text { es }\end{array}$ & $\mathrm{X}$ & $\mathrm{X}$ & $X$ & $\mathrm{X}$ & $\mathrm{X}$ & $\mathrm{X}$ & $\mathrm{X}$ & $\mathrm{X}$ \\
\hline $\begin{array}{l}\text { Napa } \\
\text { Plena } \\
\text { Flor }\end{array}$ & $X$ & & & & & & & \\
\hline
\end{tabular}

\section{B. Construcción del mapa actual}

Los procesos a considerar en el mapeo actual del proceso son los que se presentan en la figura 2 , ya que el proceso de producción de los productos seleccionados comparte estas actividades. Inicialmente se hace un mapa para cada proceso independiente y luego se hace un mapeo general. A continuación, se explica cada uno de ellos

VSM etapa de Ribera: La etapa de Ribera es la etapa en la que el cuero es preparado para ser curtido, en ella es limpiado y acondicionado asegurándole un correcto grado de humedad. Esta etapa comprende aquellos procesos que permiten la eliminación del pelo de la piel, caracterizándose por emplear grandes cantidades de agua, de lo cual deriva su nombre. La Fig. 3 muestra el mapa de cadena de valor para este proceso

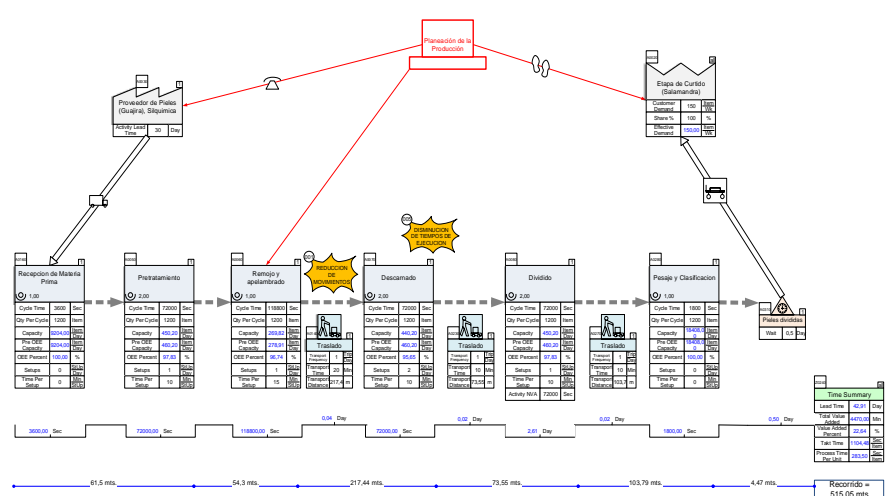

Fig. 3. Mapeo de cadena de valor actual para el proceso de Ribera

VSM Etapa de Curtido: La etapa de Curtido es la etapa que comprende las operaciones y procesos que preparan la piel para ser curtida, dar condiciones inertes y ser transformada en cuero comercial, mediante la estabilización del colágeno de la piel [24]. Para llevar a cabo el curtido, se utilizan bombos o fulones, donde se adicionan diversos químicos especiales que transforman la piel en cuero comercial. Esta etapa, también comprende la operación de rebajado y un segundo pesaje y clasificación, del cual se derivan los diversos ítems de la familia de cueros caprinos. Al finalizar esta etapa, se selecciona el producto por calidad, tamaño y condiciones específicas del cuero curtido. En la Fig.4 se exhibe el VSM actual para este proceso 


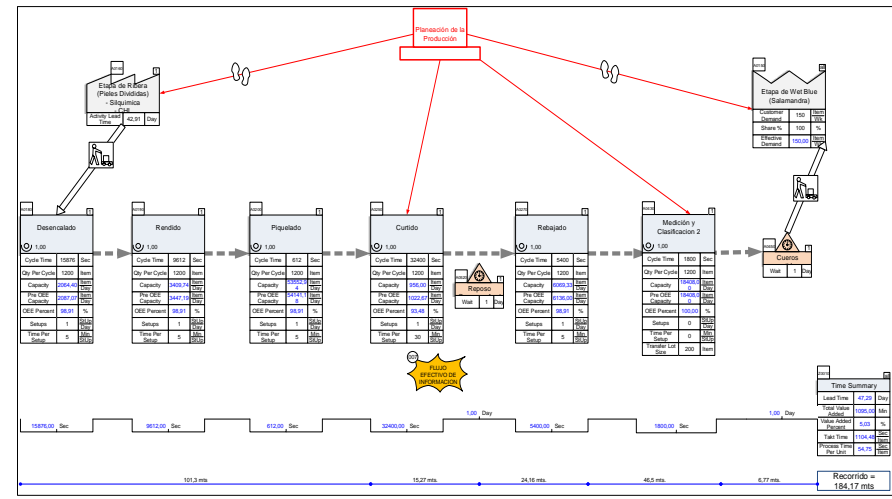

Fig. 4. Mapeo de cadena de valor actual para el proceso de curtido

VSM Etapa de Recurtido o terminado en azul: El criterio de unificar trabajos de ribera y curtido para todos los tipos de cuero y diferenciar los tipos de artículos con la etapa de recurtido y el acabado, predomina en la empresa caso de estudio y en la mayoría de las curtiembres. En esta etapa, está surgiendo el cuero que se quiere obtener al final del proceso y si presenta defectos es un buen momento para intentar corregirlos. Las operaciones que comprende esta etapa, además del Recurtido, son el Escurrido, Ablandado, Esmerilado y Templado (ver Fig. 5)

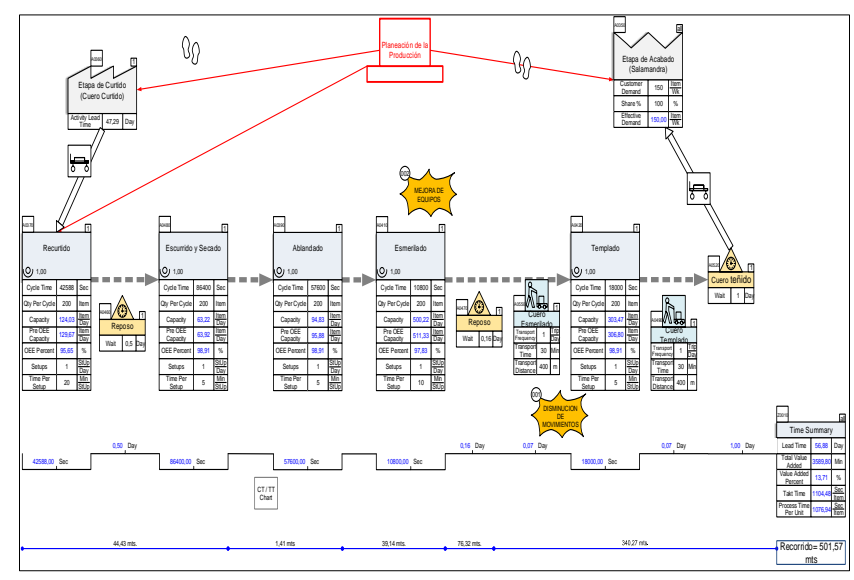

Fig 5. Mapeo de cadena de valor actual del proceso de recurtido

VSM Etapa de acabado: Comprende una serie de tratamientos a los cuales se somete la piel curtida para obtener determinadas propiedades. Estos tratamientos siempre van dirigidos para proporcionar mejoras y propiedades especiales, ya sea del lado de la flor o del lado de la carne. Con el acabado también se le proporciona al cuero protección contra los daños mecánicos, humedad, resistencia a la elaboración del artículo, suciedad; así como dar el efecto de moda deseado, como brillo, mate, doble tono, etc. Las operaciones comprendidas en esta etapa son, pintura, prensado, recorte y clasificación y medición (ver Fig.6).

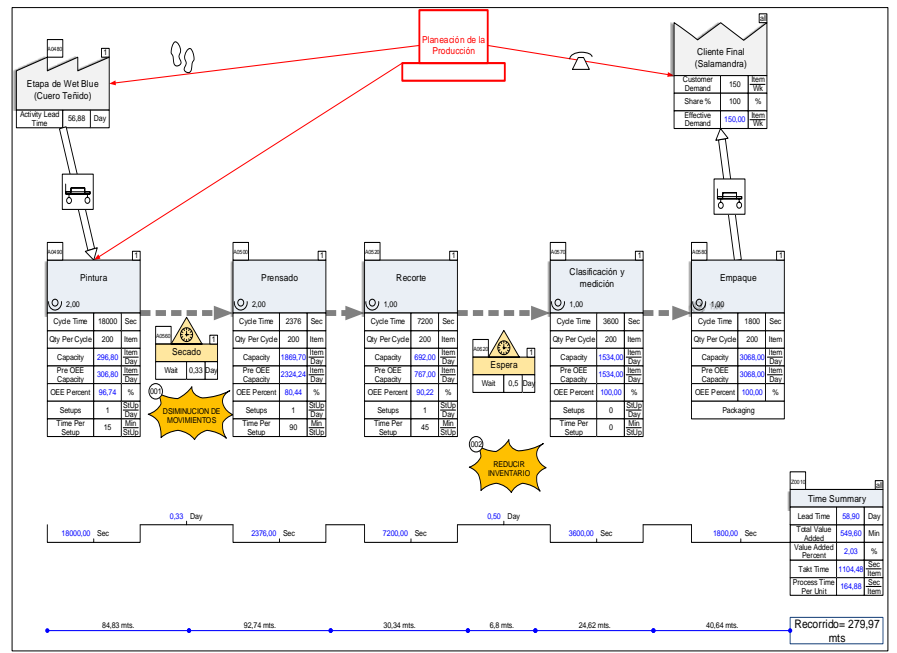

Fig. 6. Mapa de cadena de valor actual del proceso de acabado

\section{Síntesis VSM Actual de las etapas de producción}

Se analizaron en las cuatro etapas, tiempo de ciclo, porcentaje y tiempo de valor agregado y no agregado por operaciones, eficiencia de los equipos, distancias recorridas, participación de las mismas, entre otras variables, el total de todas las etapas se muestra en la tabla II.

TABLA II.

RESUltado GeNERAL De VSM En TODAs Las ETAPAS

\begin{tabular}{lcccccc}
\hline \hline \multicolumn{1}{c}{ Variable } & Resultados & Unidad & E. Ribera & E. Curtido & E. Recurtido & E. Acabado \\
\hline $\begin{array}{l}\text { Valor } \\
\text { Agregado }\end{array}$ & 9704,4 & Min & 4470 & 1095 & 3589,8 & 549,6 \\
\hline $\begin{array}{l}\text { \% del valor } \\
\text { agregado }\end{array}$ & $10.85 \%$ & $\%$ & $22,64 \%$ & $5,03 \%$ & $13,71 \%$ & $2,03 \%$ \\
\hline $\begin{array}{l}\text { Valor no } \\
\text { agregado }\end{array}$ & 11246,4 & Min & 4593,6 & 2880 & 2577,6 & 1195,2 \\
\hline $\begin{array}{l}\text { Distancia } \\
\text { Recorrida }\end{array}$ & 1480,76 & Metros & 515,05 & 184,17 & 501,57 & 279,97 \\
\hline $\begin{array}{l}\text { Número de } \\
\text { operarios }\end{array}$ & 2 & Persona & 2 & 1 & 1 & 2 \\
\hline $\begin{array}{l}\text { Tiempo de } \\
\text { ciclo }\end{array}$ & 1580,07 & Seg/ítem & 283,5 & 54,75 & 1076,94 & 164,88 \\
\hline Takt time & 1104,48 & Seg/ítem & 1104,48 & 1104,48 & 1104,48 & 1104,48 \\
\hline Lead time & 58,9 & Días & 42,91 & 47,29 & 56,88 & 58,9 \\
\hline Alistamientos & 18 & - & 5 & 5 & 5 & 3 \\
\hline $\begin{array}{l}\text { Capacidad } \\
\text { pérdida por } \\
\text { día }\end{array}$ & 348,04 & Ítem/Día & 49,09 & 781,7 & 21,82 & 539,55 \\
\hline $\begin{array}{l}\text { Capacidad } \\
\text { efectiva }\end{array}$ & 1085,78 & Ítem/Día & 29222,42 & 84460,41 & 1085,78 & 7460,7 \\
\hline \hline
\end{tabular}

El valor agregado equivale a 9704,4 minutos, es decir 21,08 días efectivos y 11245,4 minutos de tiempo que no agrega valor. El porcentaje de valor agregado de $10,85 \%$ fue calculado como el promedio de los porcentajes de cada etapa; la distancia total recorrida es de aproximadamente 1,5 kilómetros de recorrido, el tiempo de ciclo total corresponde a 1580,07 segundos por unidad equivalente a 26,33 minutos. El takt time es 1104,48 segundos. El Lead time acumulado es de 58,9 días, se efectúan 18 alistamientos que repercuten en la capacidad perdida por día equivalente a 348,04 ítems promedio por día.

Cabe resaltar el resultado obtenido de la relación del takt time y el tiempo de ciclo. Según la Fig.7, para cada una de las etapas del proceso productivo el Takt Time supera el tiempo de ciclo, lo cual denota que las operaciones inmersas en cada etapa no están generando restricciones con relación a sobreproducción, sino que por el contrario los centros de trabajo no se acercan con la mínima distancia al tiempo takt y en consecuencia de 
esto, se evidencia que estos trabajan a un ritmo mucho menor que el de la demanda de la familia de producto.

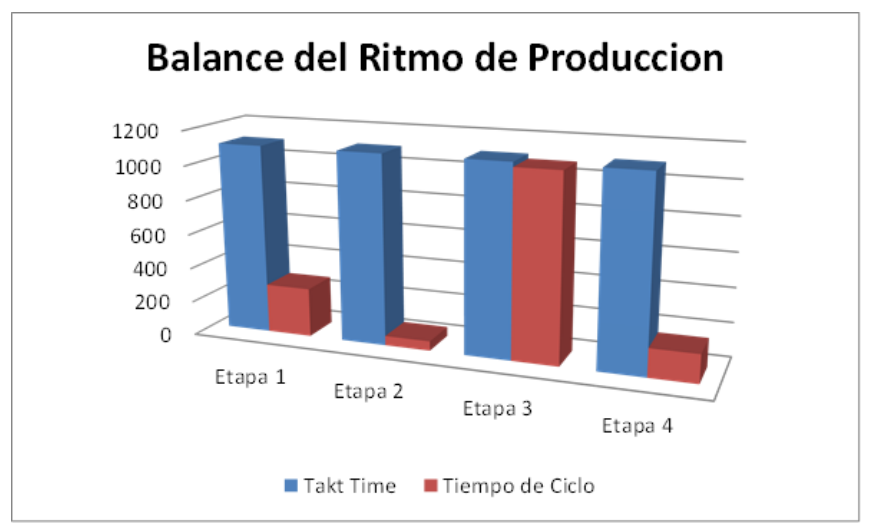

Fig 7. Relación Takt Time y Tiempo de Ciclo

\section{Identificación de oportunidades de mejora}

Para la elaboración de los VSM futuro de cada etapa fue necesario identificar y analizar los eventos kaizen que surgieron de la interpretación de cada VSM actual, teniendo en cuenta el uso de técnicas Lean y su aplicación a cada uno de los procesos (ver tabla III). Se relacionan las etapas con los eventos kaizen sugeridos para la simulación en un escenario futuro.

TABLA III.

EvENTOS KAIZEN POR ETAPA

\begin{tabular}{lcccc}
\hline \hline \multicolumn{1}{c}{ Etapa } & SMED & Layout & $\begin{array}{c}\text { Gestión } \\
\text { Visual }\end{array}$ & $5 \mathrm{~S}$ \\
\hline Ribera & $\mathrm{X}$ & $\mathrm{X}$ & & $\mathrm{X}$ \\
Curtido & & & $\mathrm{X}$ & $\mathrm{X}$ \\
Recurtido & & $\mathrm{X}$ & $\mathrm{X}$ & $\mathrm{X}$ \\
Acabado & & $\mathrm{X}$ & $\mathrm{X}$ & $\mathrm{X}$ \\
\hline \hline
\end{tabular}

A partir de esta relación de etapas con eventos kaizen, se toma como base para la simulación de alternativas, las propuestas relacionadas con rediseñar el Layout de la empresa, debido a que los problemas encontrados con mayor frecuencia a través del mapeo de cada etapa son los relacionados con movimientos innecesarios que repercuten en el Valor No Agregado en cuanto al movimiento del material en proceso, tanto dentro como fuera de las instalaciones de la empresa objeto de estudio.

\section{Simulación de propuestas de mejora}

Se plantearon tres escenarios con las mejoras previamente definidas, a continuación, se explica cada una de ellas

Escenario 1: Se propone la redistribución del espacio asignado para cada operación y como criterio de decisión, solo se tiene en cuenta el desperdicio por movimiento a nivel interno, se consideran algunas operaciones tercerizadas, se selecciona una nueva forma de distribución física y se simula el nuevo recorrido para cada etapa.

Escenario 2: en este escenario se tiene en cuenta la distribución propuesta para el escenario número uno. Adicionalmente, las operaciones tercerizadas son incluidas a nivel interno, de tal forma que se minimicen los desplazamientos fuera de la fábrica que aumenta el tiempo total de todo el proceso productivo.

Escenario 3: este escenario contempla las alternativas planteadas en el escenario uno y dos. Sumado a esto, se incluye la eliminación de operaciones que no agregan valor y de esperas innecesarias y ociosas en el proceso de fabricación.

Para la simulación tanto del estado actual como del estado futuro y el diagrama de recorrido realizado, se utilizó como herramienta el software e-VSM, versión libre 7,30. Este software vincula el programa Visio y Excel del paquete de office, creando una macro que calcula los indicadores del VSM, según la información integrada al mapeo. Así mismo, muestra las variaciones de cada estado y permite una fácil interpretación de los resultados obtenidos en cada uno.

\section{E. Construcción del mapa futuro}

Se procede a construir el mapa de cadena de valor futuro de cada uno de los cuatro procesos, incluyendo las mejoras analizadas y los escenarios descritos anteriormente. La Fig. 8 presenta los VSM Futuro para cada proceso.

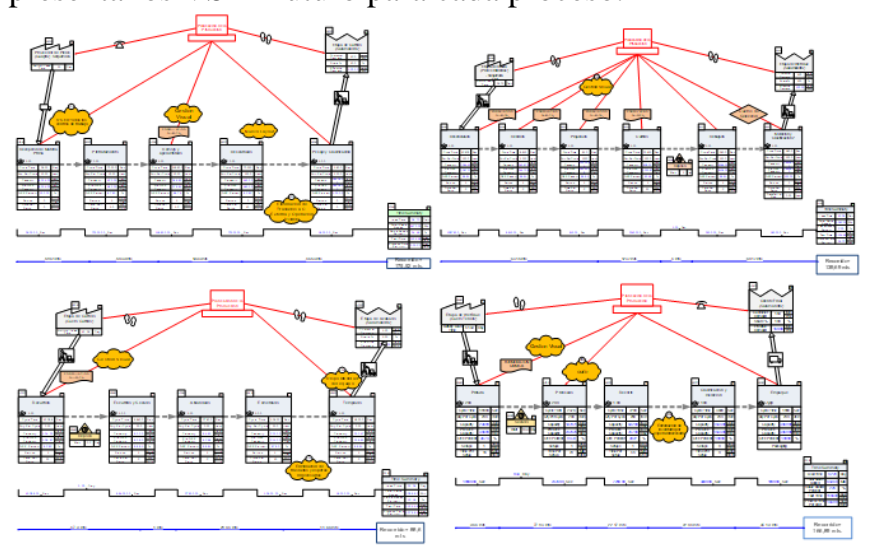

Fig. 8. VSM Futuro para los procesos de fabricación

\section{F. Selección de la estrategia de mejoramiento}

Luego de analizar los VSM futuro, se procede a identificar el resultado de la implementación de los eventos kaizen y hacer un comparativo del estado actual y el estado futuro propuesto. Para esto, se analizaron variables como: Distancia recorrida, tiempo de ciclo, valor agregado, entre otras. La tabla IV resume los principales resultados

En la tabla IV se puede observar cómo después de la implementación de las mejoras propuestas, existe un ahorro en todos los indicadores clave para los cuatros procesos de fabricación objeto de estudio, por lo que se demuestra la viabilidad del plan de acción simulado. Asimismo, en la tabla V se presenta las medidas de desempeño clave del sistema comparadas antes y después de la aplicación del VSM. Según los resultados observados en la tabla, es preciso resaltar que el valor agregado a las operaciones no tuvo ninguna variación. En este sentido, se tiene en cuenta que el proceso obedece a una estandarización, debido a los procesos químicos que incluye, por lo cual, se analizan las demás variables que corresponden a 
tiempo de valor no agregado por el desperdicio de esperas, transporte y movimientos innecesarios.

TABLA IV.

VARIABLES De COMPARACIÓN VSM ACTUAL VS VSM FUTURO

\begin{tabular}{|c|c|c|c|c|c|}
\hline Etapa & Variable & Unidad & Antes & Después & Ahorro \\
\hline \multirow[t]{7}{*}{ Ribera } & \# operaciones & - & 6 & 5 & 1 \\
\hline & $\begin{array}{l}\text { Distancia } \\
\text { recorrida }\end{array}$ & Metros & 515,05 & 175,52 & 339,53 \\
\hline & Lead Time & Días & 42,91 & 39,71 & 3,2 \\
\hline & $\begin{array}{l}\text { Tiempo de } \\
\text { ciclo }\end{array}$ & S/ítem & 283,5 & 223,5 & 60 \\
\hline & $\begin{array}{l}\text { Valor no } \\
\text { agregado }\end{array}$ & $\mathrm{S}$ & 275616 & 0 & 275616 \\
\hline & $\begin{array}{l}\text { Valor } \\
\text { Agregado }\end{array}$ & Min & 4470 & 4470 & 0 \\
\hline & $\begin{array}{l}\% \text { valor } \\
\text { agregado }\end{array}$ & $\%$ & $22.64 \%$ & $24.50 \%$ & $1.86 \%$ \\
\hline \multirow[t]{7}{*}{ Curtido } & \# operaciones & - & 5 & 6 & 0 \\
\hline & $\begin{array}{l}\text { Distancia } \\
\text { recorrida }\end{array}$ & Metros & 184,17 & 139,69 & 44,48 \\
\hline & Lead Time & Días & 47,29 & 43,09 & 4,2 \\
\hline & $\begin{array}{l}\text { Tiempo de } \\
\text { ciclo }\end{array}$ & S/ítem & 54,75 & 54,75 & 0 \\
\hline & $\begin{array}{l}\text { Valor no } \\
\text { agregado }\end{array}$ & $\mathrm{S}$ & 172800 & 86400 & 86400 \\
\hline & $\begin{array}{l}\text { Valor } \\
\text { Agregado }\end{array}$ & Min & 1095 & 1095 & 0 \\
\hline & $\begin{array}{l}\% \text { valor } \\
\text { agregado }\end{array}$ & $\%$ & $5,10 \%$ & $5,50 \%$ & $0,40 \%$ \\
\hline \multirow[t]{7}{*}{ Recurtido } & \# operaciones & - & 5 & 5 & 0 \\
\hline & $\begin{array}{l}\text { Distancia } \\
\text { recorrida }\end{array}$ & Metros & 501,57 & 88,6 & 412,97 \\
\hline & Lead Time & Días & 56,88 & 51,39 & 5,49 \\
\hline & $\begin{array}{l}\text { Tiempo de } \\
\text { ciclo }\end{array}$ & S/ítem & 1076,94 & 1076,94 & 0 \\
\hline & $\begin{array}{l}\text { Valor no } \\
\text { agregado }\end{array}$ & S & 154656 & 43200 & 111456 \\
\hline & $\begin{array}{l}\text { Valor } \\
\text { Agregado }\end{array}$ & Min & 3589,8 & 3589,8 & 0 \\
\hline & $\begin{array}{l}\% \text { valor } \\
\text { agregado }\end{array}$ & $\%$ & $13,71 \%$ & $15,18 \%$ & $1,47 \%$ \\
\hline \multirow[t]{7}{*}{ Acabado } & \# operaciones & - & 5 & 5 & 0 \\
\hline & $\begin{array}{l}\text { Distancia } \\
\text { recorrida }\end{array}$ & Metros & 279,97 & 166,89 & 113,08 \\
\hline & Lead Time & Días & 58,9 & 52,91 & 5,99 \\
\hline & $\begin{array}{l}\text { Tiempo de } \\
\text { ciclo }\end{array}$ & S/ítem & 164.88 & 164.88 & 0 \\
\hline & $\begin{array}{l}\text { Valor no } \\
\text { agregado }\end{array}$ & $\mathrm{S}$ & 71712 & 28512 & 43200 \\
\hline & $\begin{array}{l}\text { Valor } \\
\text { Agregado }\end{array}$ & Min & 549.6 & 549.6 & 0 \\
\hline & $\begin{array}{l}\% \text { valor } \\
\text { agregado }\end{array}$ & $\%$ & $2.03 \$$ & $2.26 \%$ & $0.23 \%$ \\
\hline
\end{tabular}

TABLA V.

COMPARACIÓN GENERAL VSM ACTUAL VS VSM FUTURO

\begin{tabular}{cccc}
\hline \hline Variable & VSM actual & VSM Futuro & Ahorro \\
\hline $\begin{array}{c}\text { Distancia } \\
\text { recorrida (m) }\end{array}$ & 1480,76 & 570,7 & 910,06 \\
\hline $\begin{array}{c}\text { Tiempo de ciclo } \\
\text { (seg) }\end{array}$ & 1580,07 & 1520,07 & 60 \\
\hline $\begin{array}{c}\text { Lead time } \\
\text { (días) }\end{array}$ & 58,9 & 52,91 & 5,99 \\
\hline $\begin{array}{c}\text { Valor agregado } \\
\text { (min) }\end{array}$ & 9704,4 & 9704,4 & 0 \\
\hline $\begin{array}{c}\text { Valor no } \\
\text { agregado (seg) }\end{array}$ & 674784 & 158112 & 516672 \\
\hline \hline
\end{tabular}

La variable que refleja una mejora considerable es la distancia recorrida, resultante de las variaciones en el layout de la fábrica, el VSM futuro refleja que el ahorro en desplazamientos a través de todo el proceso productivo es de 910 metros.
Tomando como referente el estudio realizado por Delgado \& Torres (2001), el tiempo de ciclo promedio para todos los empresarios del cuero es de 21 días. Por lo tanto, en el transcurso de este proyecto se pretendia disminuir el tiempo de ciclo a ese valor meta o por lo menos acercarlo al promedio del tiempo de ciclo del sector. Conforme a lo anterior, la tabla 5 muestra que el tiempo de ciclo por unidad paso de 1580,07 segundos a 1520,07 segundos, equivalentes a aproximadamente 21 días por el lote de fabricación. El Lead time disminuyo en 5,99 días, pasando de 58,9 días a 52,91 días, y el valor no agregado disminuyo al eliminar traslados y esperas que no agregaban valor, lo cual es beneficioso tanto para la empresa como para el cliente final

\section{CONCLUSIONES}

El mapeo de la cadena de valor es una herramienta primordial, para el diagnóstico, análisis y proyección de estados futuros ante sistemas productivos reales. Basando su aplicación en la familia de productos más representativa, con el fin de conocer y comprender los flujos de información, tiempo, materiales, inventario, desperdicios y actividades que no agregan valor, como son las esperas y desplazamientos innecesarios.

Con respecto al sector de curtiembres, la aplicación de la metodología se torna más compleja por el tipo de producto que se está fabricando, ya que, al ser el cuero un producto de altos requerimientos de calidad, es la producción por etapas lo que facilita que cada proceso se realice de la manera adecuada. Por tal razón, fue necesaria la realización del VSM por etapas de producto, con el fin de abordar cada una de ellas y generar ideas de mejora aplicables a pequeña escala con el fin de analizar detenidamente la problemática particular de cada etapa y posteriormente integrar las ideas de mejora en pro de evaluar el desempeño de un estado futuro a nivel de todo el proceso productivo.

El VSM actual permitió plantear y mapear una situación futura, teniendo en cuenta la subutilización de los espacios en fábrica, los tiempos perdidos por desplazamientos y movimientos innecesarios. Además, a partir de este se generaron tres escenarios en los cuales también se tuvo en cuenta el costo por unidad de cada producto.

Los eventos Kaizen se enfocaron en la disminución de movimientos y redistribución del Layout de la fábrica. Además, se tuvo en cuenta proponer mejoras encaminadas a establecer una comunicación efectiva a través de la Gestión visual, y la disminución de alistamientos en algunas operaciones puntuales a través de SMED, también se sugirió la implementación de 5'S como herramienta para la disminución de desperdicios y la persecución del mejoramiento continuo en la empresa.

\section{REFERENCIAS}

M. Nguyen and N. Do, "Re-engineering Assembly line with Lean Techniques," Procedia CIRP, vol. 40, pp. 590-595, 2016, DOI: 10.1016/j.procir.2016.01.139.

[2] I. Serrano, Análisis de la aplicabilidad de la técnica Value Stream 
Mapping en el rediseño de sistemas productivos. 2007.

[3] M. Villadiego, "Diseño metodológico para la implementación del Value Stream Mapping en una empresa manufacturerea colombiana dedicada al mercado de ascensores," Universidad NAcional de Colombia Sede Medellín, 2012.

[4] J. P. Womack and D. T. Jones, Lean thinking: Banish waste and create walth in your corporation. 2012.

[5] I. Serrano, C. Ochoa, and R. de Castro, "An evaluation of the value stream mapping tool," Bus. Process Manag. J., vol. 14, no. 1, pp. 3952, 2008, DOI: $10.1108 / 14637150810849391$.

[6] P. M. Masuti and U. A. Dabade, "Lean manufacturing implementation using value stream mapping at excavator manufacturing company," Mater. Today Proc., vol. 19, pp. 606-610, 2019, DOI: 10.1016/j.matpr.2019.07.740.

[7] Z. Huang, J. Kim, A. Sadri, S. Dowey, and M. S. Dargusch, "Industry 4.0 : Development of a multi-agent system for dynamic value stream mapping in SMEs," J. Manuf. Syst., vol. 52, pp. 1-12, 2019, DOI: 10.1016/j.jmsy.2019.05.001

[8] D. Knoll, G. Reinhart, and M. Prüglmeier, "Enabling value stream mapping for internal logistics using multidimensional process mining," Expert Syst. Appl., vol. 124, pp. 130-142, 2019, DOI: 10.1016/j.eswa.2019.01.026.

[9] J. Wesana, X. Gellynck, M. K. Dora, D. Pearce, and H. De Steur, "Measuring food and nutritional losses through value stream mapping along the dairy value chain in Uganda," Resour. Conserv. Recycl., vol. 150, 2019, DOI: 10.1016/j.resconrec.2019.104416.

[10] A. Deshkar, S. Kamle, J. Giri, and V. Korde, "Design and evaluation of Lean Manufacturing Framework using Value Stream Mapping ( VSM ) for a plastic bag manufacturing unit.," Mater. Today Proc., vol. 5, no. 2, pp. 7668-7677, 2018, DOI: 10.1016/j.matpr.2017.11.442.

[11] A.-A. Dadashnejad and C. Valmohammadi, "Investigating the effect of value stream mapping on overall equipment effectiveness: a case study," Total Qual. Manag. Bus. Excell., vol. 30, no. 3-4, pp. 466482, 2017, DOI: 10.1080/14783363.2017.1308821.

[12] G. A. Marodin, A. G. Frank, G. L. Tortorella, and D. C. Fetterman, "Lean production and operational performance in the Brazilian automotive supply chain," Total Qual. Manag. Bus. Excell., vol. 30, no. 3-4, pp. 370-385, 2017, DOI: 10.1080/14783363.2017.1308221.

[13] K. Venkataraman, B. V. Ramnath, V. M. Kumar, and C. Elanchezhian, "Application of Value Stream Mapping for Reduction of Cycle Time in a Machining Process," Procedia Mater. Sci., vol. 6, no. Icmpc, pp. 1187-1196, 2014, DOI: 10.1016/j.mspro.2014.07.192.

[14] A. R. Rahani and M. Al-Ashraf, "Production Flow Analysis through Value Stream Mapping: A Lean Manufacturing Process Case Study," Procedia Eng., vol. 41, no. Iris, pp. 1727-1734, 2012, DOI: 10.1016/j.proeng.2012.07.375.

[15] W. Faulkner and F. Badurdeen, "Sustainable Value Stream Mapping (Sus-VSM): Methodology to visualize and assess manufacturing sustainability performance," J. Clean. Prod., vol. 85, pp. 8-18, 2014, DOI: 10.1016/j.jclepro.2014.05.042.

[16] A. Brown, J. Amundson, and F. Badurdeen, "Sustainable value stream mapping (Sus-VSM) in different manufacturing system configurations: application case studies," J. Clean. Prod., vol. 85, pp. 164-179, Jun. 2014, DOI: 10.1016/j.jclepro.2014.05.101.

[17] B. K. Jeong and T. E. Yoon, "Improving it process management through value stream mapping approach: a case study," J. Inf. Syst. Technol. Manag., vol. 13, no. 3, pp. 389-404, 2016, DOI: 10.4301/S1807-17752016000300002.

[18] D. Stadnicka and R. M. Chandima Ratyanake, "Minimization of service disturbance: VSM based case study in telecommunication industry," IFAC-PapersOnLine, vol. 49, no. 12, pp. 255-260, 2016, DOI: 10.1016/j.ifacol.2016.07.609.

[19] O. Delgado and M. Torres, "Caracterización de las microempresas pertenecientes al subsector cuero en el municipio de 'El Cerrito' Valle del Cauca." Guadalajara de Buga - Colombia, 2001.

[20] M. Nash and S. Poling, Mapping the total value stream. New York, 2008.

[21] M. Rother and J. Shook, "Learning to See Value Stream Mapping to Create Value and Eliminate Muda," Lean Enterp. Inst. Brookline, p. 102, 2003, DOI: $10.1109 / 6.490058$.

[22] J. L. Burbidge, "Production flow analysis for planning group technology," J. Oper. Mana, vol. 10, no. 1, pp. 5-27, 1991.

[23] P. Hines, N. Rich, and A. E. Esain, "Value stream mapping: A distribution industry application Benchmarking," An Int. J., vol. 6, no. 1 , pp. $60-77,1999$, DOI: $10.1108 / 14635779910258157$.

[24] A. Pinzón, N. Morales, D. Rodríguez, J. Saavedra, and C. Alarcón, Guía de producción más limpia para el sector curtiembres de Bogotá. Enfoque en vertimientos y residuos. Bogotá - Colombia, 2015.

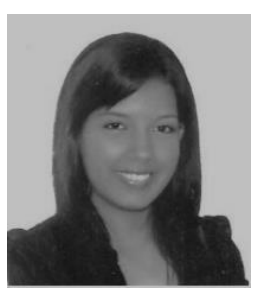

M. Bedoya-Diaz, nacida el 14 de enero de 1993, Guadalajara de Buga, Valle del Cauca, Colombia. Ingeniera Industrial, de la Universidad del Valle, Buga, Valle del Cauca, Colombia. ORCID: https://orcid.org/0000-0002-91255564

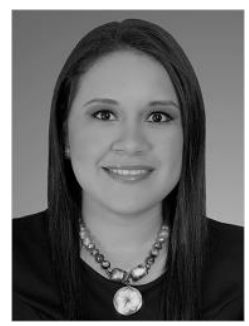

V.L. Chud-Pantoja, nacida el 3 de diciembre de 1988, Cali, Valle del Cauca, Colombia. Ingeniera Industrial, Magister en Ingeniería Industrial de la Universidad del Valle, Santiago de Cali, Valle del Cauca, Colombia. Actualmente Docente tiempo completo del área de Ingeniería Industrial en la Universidad del Valle Sede Zarzal, Valle del Cauca. Directora del Grupo de Investigación GEDESC.

ORCID: https://orcid.org/0000-0001-8842-637X

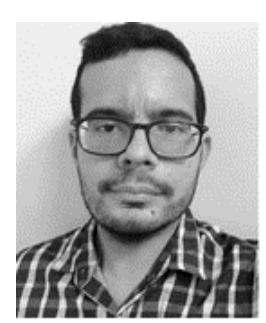

A.M. Paredes-Rodríguez, nacido el 25 de febrero de 1994 en San Pedro, Valle del Cauca, Colombia. Ingeniero Industrial, Magister en Ingeniería Industrial de la Universidad del Valle, Valle del Cauca, Colombia. Docente tiempo completo del Programa de Ingeniería Industrial de la Corporación Universitaria Minuto de Dios, Buga, Valle del Cauca. Sublíder del grupo de investigación GICIDET. Líder del semillero de investigación GLIOSP.

ORCID: http://orcid.org/0000-0001-9196-9965 